\title{
Ductal carcinoma in situ (DCIS) in breast fibroadenoma
}

\author{
Manar El-Essawy ${ }^{1 *}$ D, Amal AL Haidary ${ }^{1}$ and Abdul Latif Khan²
}

\begin{abstract}
Background: Fibroadenoma is the most common benign tumor; although malignant, changes are rare. Any changes or increase in size should be evaluated thoroughly to exclude any malignant formation.

Case presentation: A 25-year-old female presenting to our department complaining of enlargement of the previously stable mass underwent imaging assessment and biopsy which revealed ductal carcinoma in situ changes developed in a fibroadenoma.

Conclusion: Although malignant changes in a fibroadenoma are rare, biopsy of increasing size fibroadenoma is advised to avoid missing any malignant changes; the incidence is more in the complex fibroadenoma.
\end{abstract}

Keywords: DCIS, Fibroadenoma, Breast cancer

\section{Background}

Fibroadenoma is the most common benign breast neoplasm seen in young women. Although it is benign, there is a very rare risk to develop malignancy; this risk is more evident in complex fibroadenomas and those with a family history of breast cancer. Most of the cases of cancer within fibroadenoma are diagnosed incidentally during a pathological examination. We diagnosed a case of ductal carcinoma in situ which developed within fibroadenoma that showed a recent increase in size after a long time of stability that warrants biopsy which revealed malignant changes; the patient was seen by breast surgeon that decided to do a lumpectomy.

\section{Case Presentation}

A 25-year-old female is frequently coming to our department to follow up multiple bilateral breast masses by ultrasound; these masses are stable throughout a period of 4 years and appeared

\footnotetext{
*Correspondence: ELESSAWYMA@ngha.med.sa

'Department of Radiology, National Guard Health Affairs, King Abdulaziz Medical City, Riyadh, Saudi Arabia

Full list of author information is available at the end of the article
}

circumscribed, transversely oriented, hypoechoic benign-looking, and diagnosed as multiple fibroadenomas. Since the masses were stable for more than 2 years, the case was coded as BIRADS 2. Recently, the patient presented with a 1-month history of increased size of one of the left breast lesions. Ultrasound was done that revealed an increase in the size of the lesion of patient's concern. This mass appeared homogenous hypoechoic with few foci of calcification and demonstrated mild irregularity of its margin (Fig. 1). The mass showed increased vascularity in color Doppler study, and it measured $3.3 \times 2.5 \mathrm{~cm}$. It is located at the inner aspect of the left breast opposite 9 o'clock. Core biopsy was done using a 14-gauge needle (Fig. 2) revealing ductal carcinoma in situ arising in fibroadenoma of low to intermediate nuclear grade and cribriform solid architecture (Fig. 3), PR and ER are negative. This case is the first case diagnosed as DCIS within fibroadenoma in our hospital. The patient has no family history of breast cancer, no skin changes, pain, or nipple discharge; her grandmother had ovarian carcinoma.

The patient then called back to do mammogram and MRI for the evaluation of other masses and to 


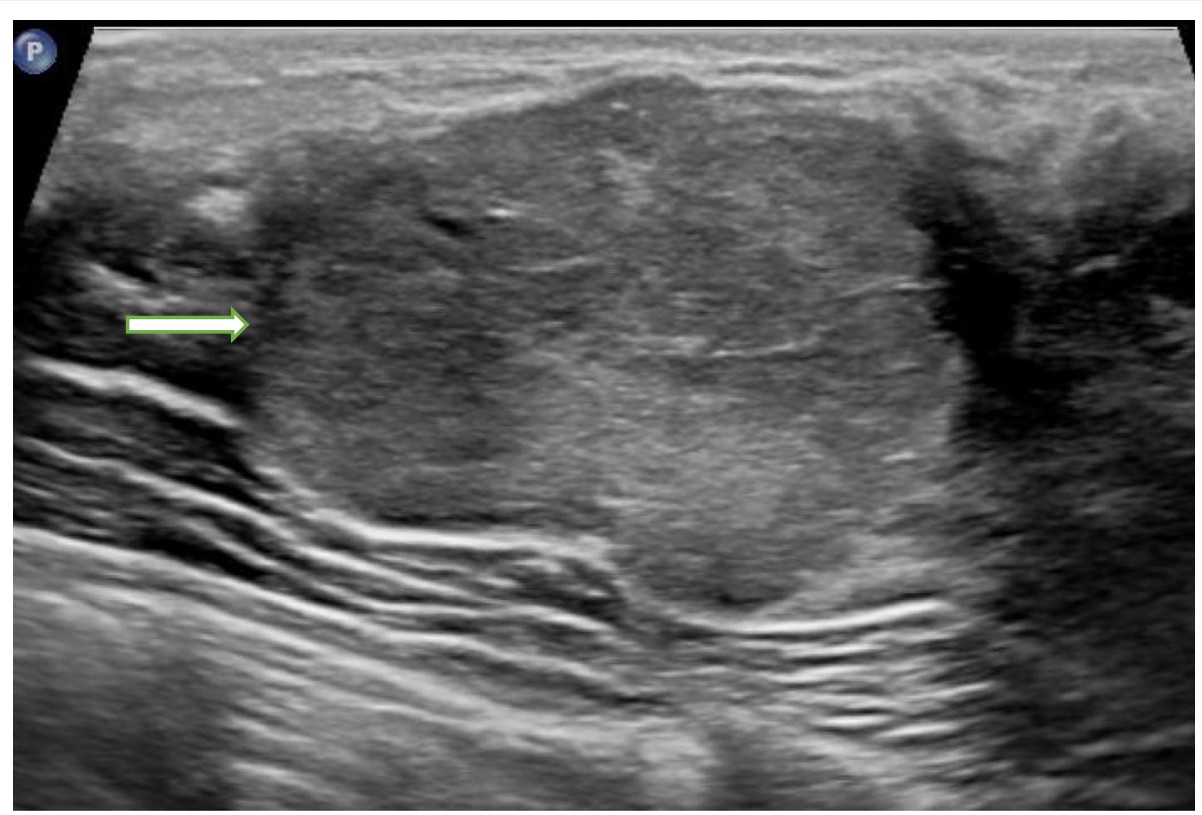

Fig. 1 A 25-year-old female with DCIS in fibroadenoma. Ultrasound examination of the left breast demonstrates an irregular hypoechoic deeply seated mass lesion (arrow) in its inner aspect opposite 9 o'clock with irregular indistinct margin (Department of Radiology, King Abdulaziz Medical City, Riyadh, Saudi Arabia, 2020)

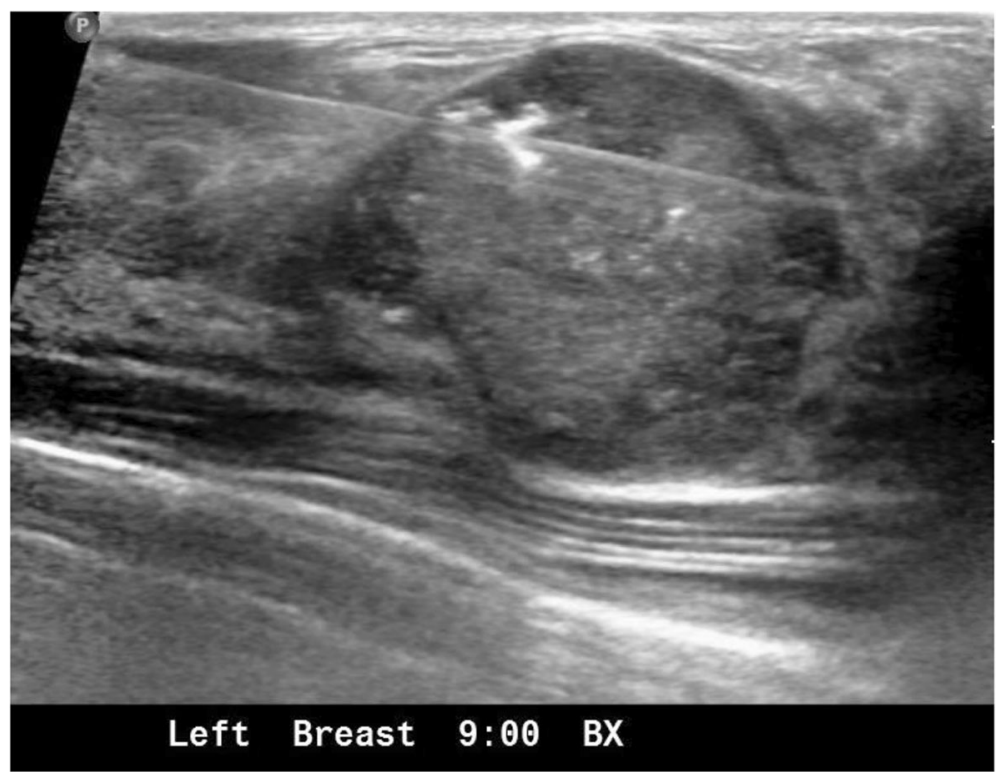

Fig. 2 A 25-year-old female with DCIS in fibroadenoma. Ultrasound-guided biopsy using 14-gauge core needle that proved the diagnosis (Department of Radiology, King Abdulaziz Medical City, Riyadh, Saudi Arabia, 2020) 


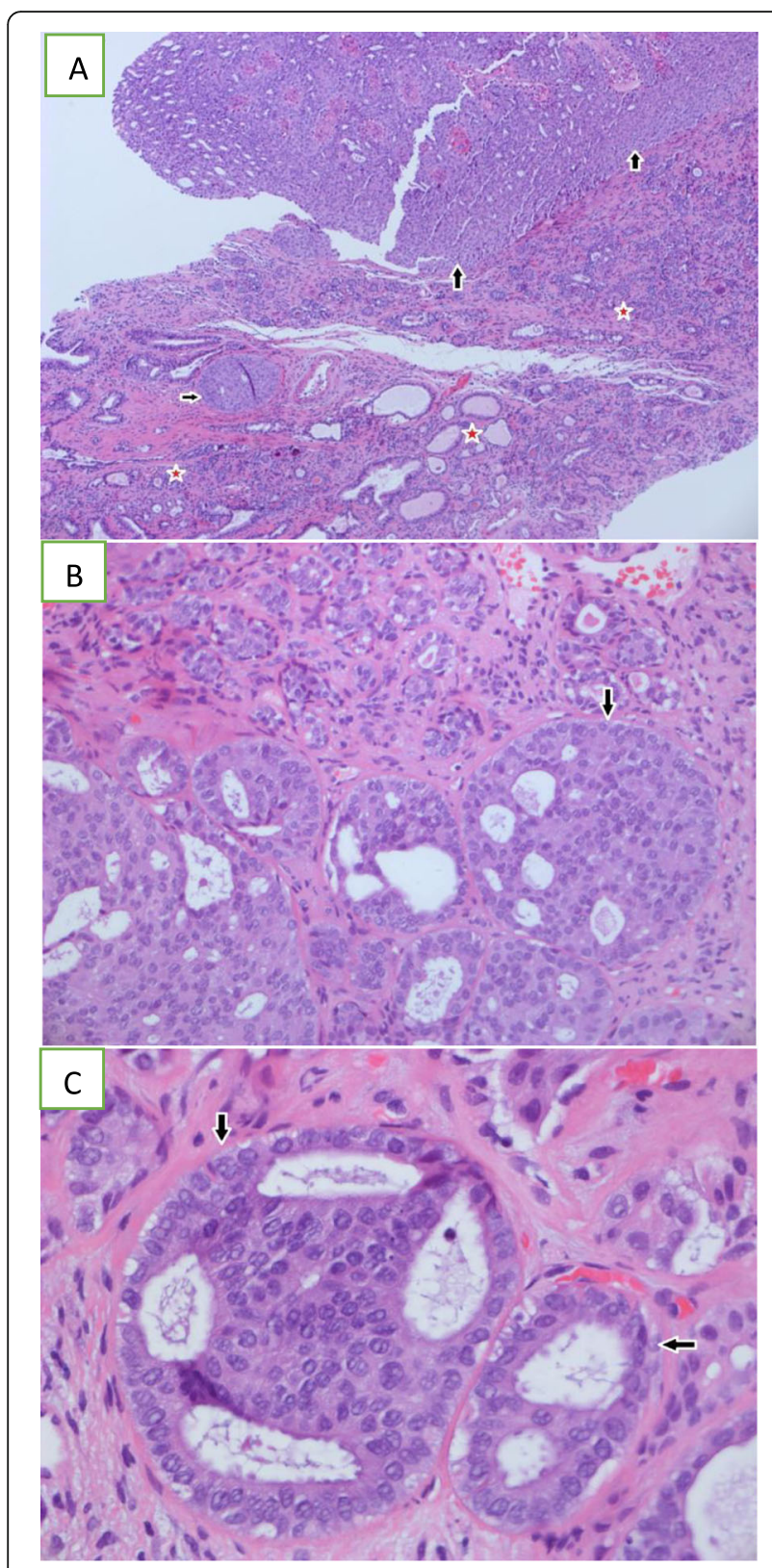

Fig. 3 Histopathology images of DCIS in fibroadenoma. a-c Hematoxylin and Eosin -stained sections of fibroadenoma (star) with areas of DCIS (arrow) (Department of Radiology, King Abdulaziz Medical City, Riyadh, Saudi Arabia, 2020)

detect any suspicious microcalcification. The mammogram was very dense (Fig. 4); scattered foci of microcalcification are noted, and the mass could not be identified. In post-contrast breast MRI, the concerned mass exhibited low signal intensity on T2WI (Fig. 5) and demonstrated a rapid enhancement in early post-contrast series (Fig. 6) with washout of the peripheral part of the lesion compatible with the areas of DCIS and progressive enhancement of the central portion that could be a part of fibroadenoma in the delayed series (Fig. 7). Type III dynamic curve is identified in the suspicious area (Fig. 8). The patient was seen by a breast surgeon that asked for a biopsy of the other masses that was done and revealed benign lesions. The decision was made for a lumpectomy.

\section{Discussion}

Fibroadenoma is a benign fibroepithelial tumor common in young women; the overall incidence is about $2.2 \%$; they represent about $68 \%$ of breast masses, and $70 \%$ presented as a single mass and $10-25 \%$ as multiple masses [1]. It is composed of connective and epithelial tissues [2].

Although there is no risk related with fibroadenoma to breast cancer, very rare malignant changes can develop within the fibroadenoma [3]. Some studies have revealed that malignant transformation is seen more in complex fibroadenoma than simple one whereas another study reported that the risk is highly associated with the percentage of epithelial proliferation within the fibroadenoma rather than the presence of fibroadenoma itself [4]. Because the malignancy is rare in fibroadenoma, conservative management with follow-up is accepted except if the mass has progressed in size [5].

The incidence of a carcinoma evolving within a fibroadenoma represents 0.002 to $0.0125 \%$, in which only $20 \%$ of these were DCIS [2], other malignancies as lobular carcinoma in situ; invasive ductal and lobular carcinomas are also seen [6, 7]. In our case, the case is considered as simple fibroadenoma with DCIS developed on top.

A recent publication considers a woman with complex fibroadenoma in the presence of a family history of breast cancer as a risk factor that should start mammographic screening at an early age [8].

The management of fibroadenoma differs according to the age of the patient. Those below 35, if the regression of the mass is noted, should consider further follow-up until the mass regressed and disappear completely. If the mass is stable by the age of 35 after 6-12 months follow-up, then excision is advised. On the other hand, if the mass has increased, immediate surgical excision is performed [9].

If the age is above 35 with a stable or increased size of fibroadenoma after a follow-up of 6-12 months, excision is advised; however, the complex fibroadenoma should be surgically removed [10]. 


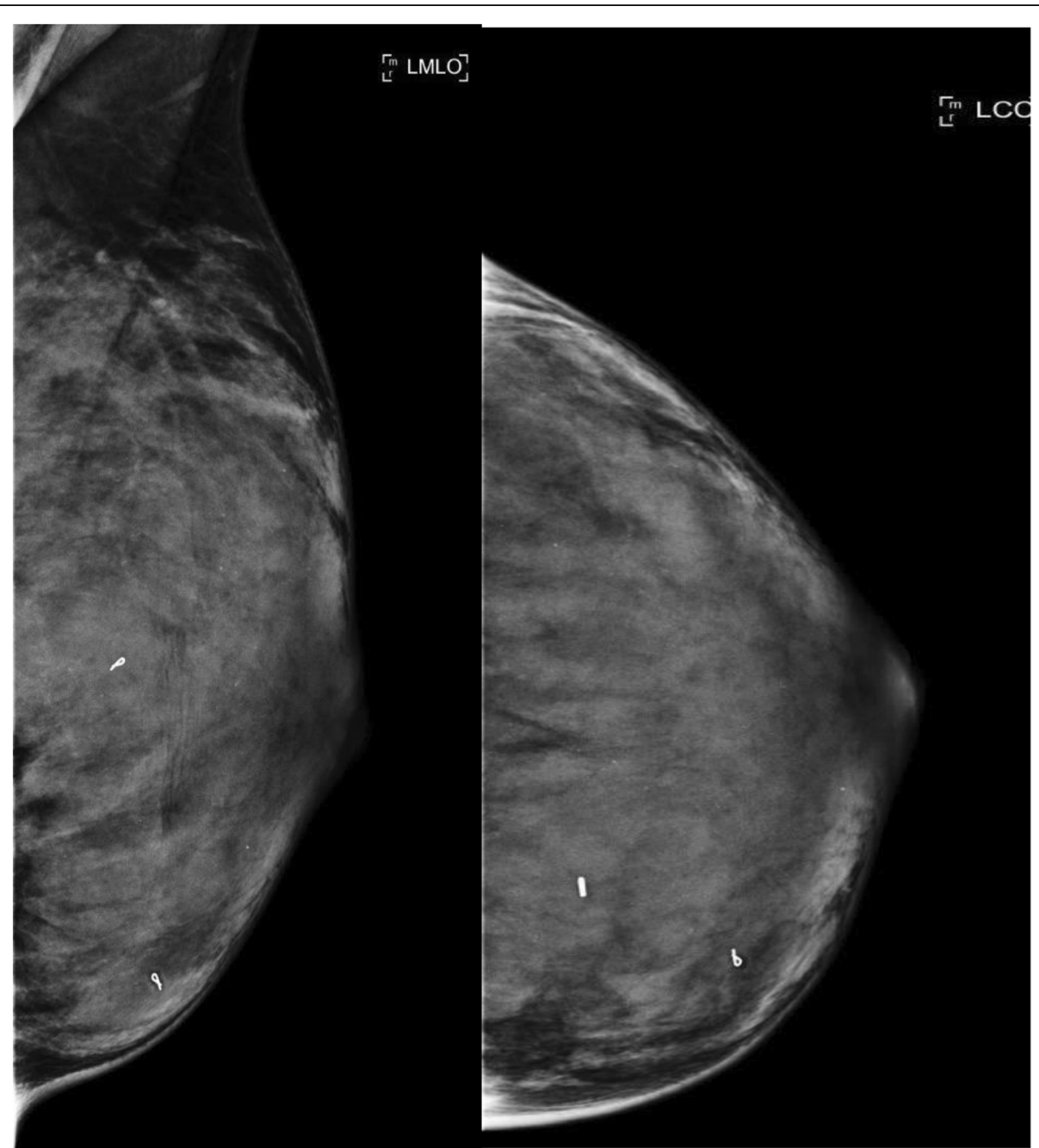

Fig. 4 A 25-year-old female with DCIS in fibroadenoma. Mammogram MLO and CC views of the left breast are extremely dense (ACR D), no discrete masses could be seen, and scattered microcalcification is noted. Tissue markers of previous ultrasound-guided biopsies are noted in the inner lower and inner middle part of the breast (Department of Radiology, King Abdulaziz Medical City, Riyadh, Saudi Arabia, 2020)

Our case referred to a breast surgeon that decided surgical removal of the mass.

The imaging findings of carcinoma arising from a fibroadenoma were reported in few studies that demonstrate a non-circumscribe indistinct margins, and clustered microcalcifications within the mass in mammogram should raise the suspicion of malignancy while in ultrasound, a focal increase of color flow signals within the mass on color Doppler study or increase size raise suspicion of malignancy [11]. In our case, the mammogram is markedly dense, and the mass cannot be appreciated. Scattered microcalcification is noted, with no suspicious microcalcification at the anticipated site of the mass. The alarming signs were demonstrated in ultrasound that identifies the enlargement of the mass and the irregular appearance of the margin.

Typically, on MRI, fibroadenoma appears as a round or oval-shaped mass with a smooth margin. Fibroadenoma can exhibit variable enhancement; however, enhancement usually persists until the delayed phase [12]. Few reports described dynamic contrast-enhanced breast MRI (DCE-b MRI) findings of carcinoma within fibroadenoma as a mass showing a pattern of early peak enhancement in the central area and delayed rim enhancement in the periphery, and in histology, DCIS was found in the center of the fibroadenoma [13]. Others described that the area of DCIS within the fibroadenoma showed rapid initial enhancement 


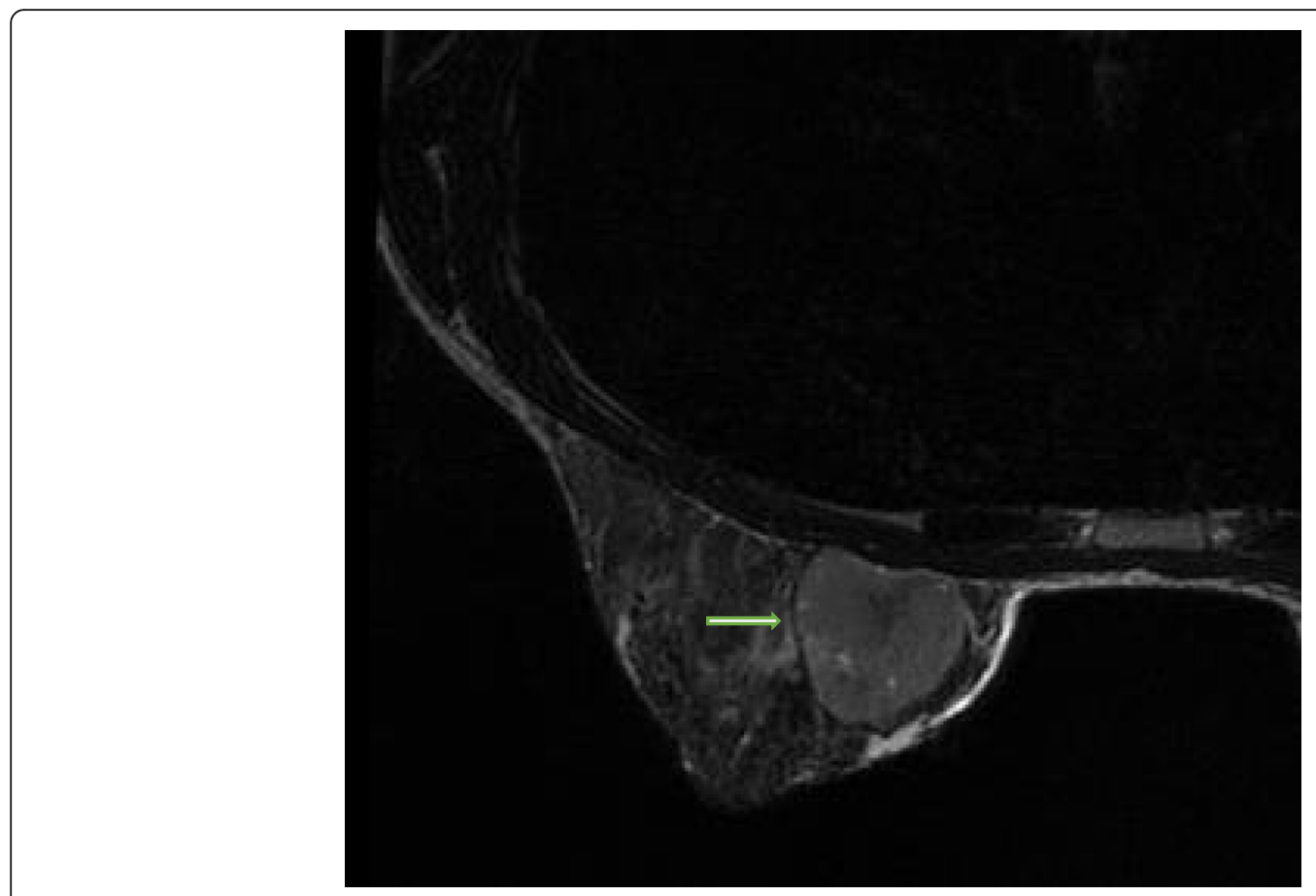

Fig. 5 A 25-year-old female with DCIS in fibroadenoma. DCE-b MRI T2WI showed a deeply located low signal intensity mass in the inner aspect left breast (arrow) (Department of Radiology, King Abdulaziz Medical City, Riyadh, Saudi Arabia, 2020)

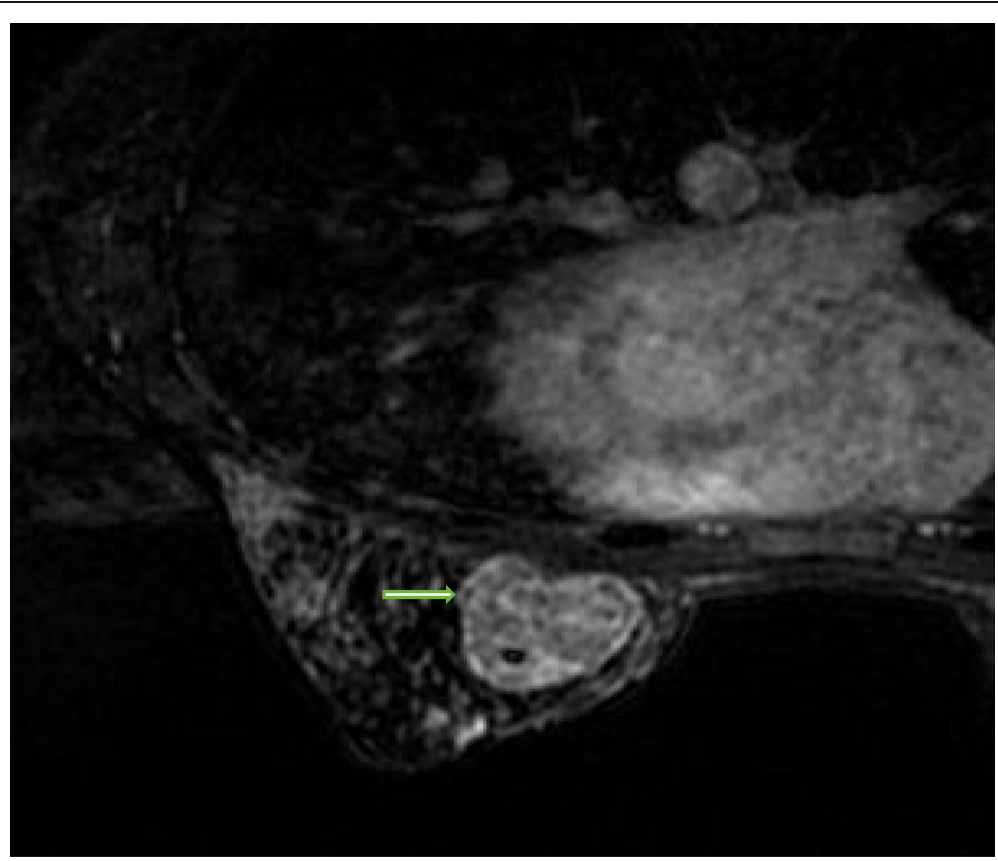

Fig. 6 A 25-year-old female with DCIS in fibroadenoma. Post-contrast DCE-b MRI early phase showed rapid heterogenous enhancement of the mass (arrow), tissue marker noted within (Department of Radiology, King Abdulaziz Medical City, Riyadh, Saudi Arabia, 2020) 


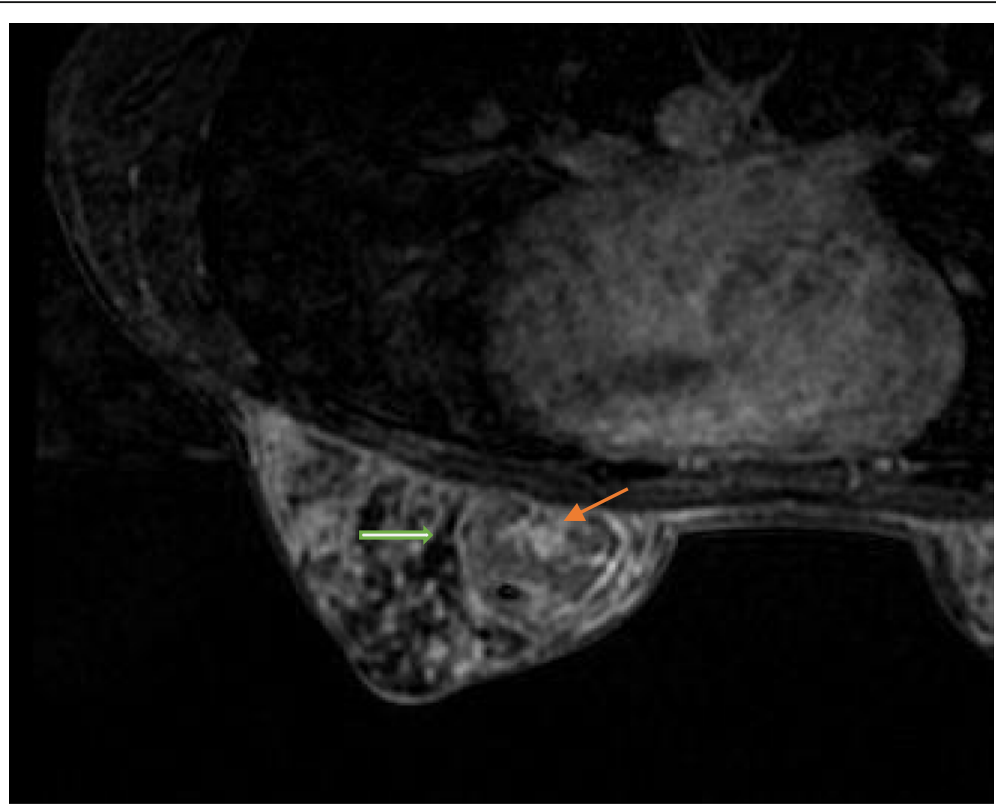

Fig. 7 A 25-year-old female with DCIS in fibroadenoma. Post-contrast DCE-b MRI late phase showed washout (thick green arrow) of the periphery of the lesion that represents DCIS, and progressive enhancement of the central portion (orange thin arrow) could be a part of fibroadenoma (Department of Radiology, King Abdulaziz Medical City, Riyadh, Saudi Arabia, 2020)

and washout kinetics on DCE-b MRI, whereas the major part of the mass was consistent with fibroadenoma [12]. In our case, DCE-b MRI demonstrates early rapid and delayed washout enhancement in the majority of the mass and progressive enhancement of its central portion which might represent a remaining part of fibroadenoma.

\section{Conclusion}

Although fibroadenoma is a benign lesion, it rarely can harbor a malignant cell; thus, follow-up is advised, and biopsy and/or excision if any progressive changes is seen. Multifocality does not always equal benignity, and each lesion should be assessed separately to identify potential breast cancer and/or highrisk lesions.

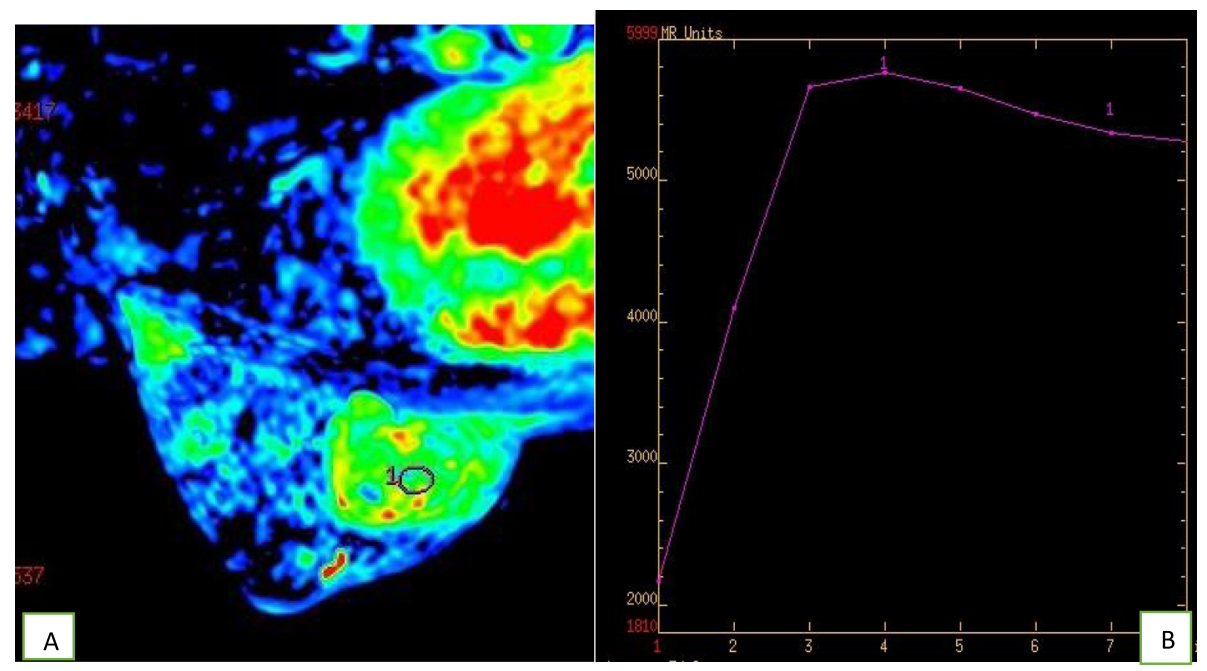

Fig. 8 a, b A 25-year-old female with DCIS in fibroadenoma. Color-coded and dynamic curve in DCE-b MRI show areas of rapid enhancement with washout compatible with type III suspicious dynamic curve (Department of Radiology, King Abdulaziz Medical City, Riyadh, Saudi Arabia, 2020) 


\section{Abbreviations}

DCE-b MRI: Dynamic contrast enhancement breast magnetic resonance imaging; DCIS: Ductal carcinoma in situ; ER: Estrogen receptor; PR: Progesterone receptor; T2Wl: T2-weighted images

\section{Acknowledgements}

NA.

\section{Authors' contributions}

ME is the correspondence author, designed and revised the work, interpreted the data, and submitted the case. ME has approved the submitted version for publication. AA has drafted the work and approved the submitted version for publication. AK has provided and interpreted the pathology images and approved the submitted version for publication. No disclosure. All authors read and approved the final manuscript.

\section{Funding}

No funds.

\section{Availability of data and materials}

The case and the images are available from the Radiology Department, Women's Imaging Section in National Guard Hospital, Riyadh, Kingdom of Saudi Arabia

\section{Ethics approval and consent to participate}

Ethics approval of the patient's consent for publication in the case report is waived in our NGHA organization. We had only a consent form for biopsy. A written approval was obtained from the participant.

\section{Consent for publication}

The authors consented for the submission of the manuscript and publication. I got written approvals from the authors to publish. The patient included in this research gave written informed consent to publish the data contained within this study. The authors disclosed no competing interests and no relevant relationships.

\section{Competing interests}

The authors declare that they have no competing interests.

\section{Author details}

${ }^{1}$ Department of Radiology, National Guard Health Affairs, King Abdulaziz Medical City, Riyadh, Saudi Arabia. ²Department of Pathology, National Guard Health Affairs, King Abdulaziz Medical City, Riyadh, Saudi Arabia.

Received: 14 January 2020 Accepted: 23 April 2020

Published online: 06 May 2020

\section{References}

1. Lee M, Soltanian HT (2015) Breast fibroadenomas in adolescents: current perspectives. Adolesc. Health Med. Ther. 6:159-163 Published 2015 Sep 2

2. Greenberg R, Skornick Y, Kaplan O (1998) Management of breast fibroadenomas. J. Gen. Intern. Med. 13:640-645

3. Shaik A, Ruterbusch J, Abdulfatah E, Shrestha R, Daaboul M, Pardeshi V et al (2018) Breast fibroadenomas are not associated with increased breast cancer risk in an African American contemporary cohort of women with benign breast disease. Breast Cancer Res. 20(1):91

4. Dupont WD, Page DL, Parl FF, Vnencak-Jones CL, Plummer WD Jr, Rados MS et al (1994) Long-term risk of breast cancer in women with fibroadenoma. N. Engl. J. Med. 331:10-15

5. Nassar A, Visscher DW, Degnim AC et al (2015) Complex fibroadenoma and breast cancer risk: a Mayo Clinic benign breast disease cohort study. Breast Cancer Res. Treat. 153(2):397-405

6. Kuijper A, Preisler-Adams SS, Rahusen FD, Gille JJ, van der Wall E, van Diest PJ (2002) Multiple fibroadenomas harboring carcinoma in situ in a woman with a family history of breast/ovarian cancer. J. Clin. Pathol. 55:795-797

7. Aydin OU, Soylu L, Ercan A, Bilezikçi B, Koçak S (2015) Invasive ductal carcinoma developing from fibroadenoma. J Breast Health 11(4):195-198

8. Saadallah F, Bouraoui I, Naija L, Sakhri S, Zemni I, Hassouna JB, Dhieb TB, Bouzaiene H, Rahal K (2019) Coexistence of invasive ductal breast carcinoma and fibroadenoma. Pan Afr Med J 33
9. Wu YT, Chen ST, Chen CJ, Kuo YL, Tseng LM, Chen DR et al (2014) Breast cancer arising within fibroadenoma: collective analysis of case reports in the literature and hints on treatment policy. World J Surg Oncol. 12:335

10. Gollapalli V, Bibi AK, Shah S, Gilchrist B (2017) DCIS breast arising in a fibroadenoma- case report of a rare condition. Clin Surg. 2:1312

11. Park EK, Cho KR, Seo BK, Woo OH, Hyeon J, Song SE, Bae JW (2015) Radiologic findings of ductal carcinoma in situ arising within a juvenile fibroadenoma: mammographic, sonographic and dynamic contrastenhanced breast MRI feature. Iran. J. Radiol. 12(2):e17916

12. Kato F, Omatsu T, Matsumura W, Takahashi M, Hosoda M, Takahashi $\mathrm{H}$ et al (2011) Dynamic MR findings of ductal carcinoma in situ within a fibroadenoma. Magn. Reson. Med. Sci. 10(2):129-132

13. Shin JH, Choi HY, Lee SN, Kim YJ (2006) Microinvasive ductal carcinoma arising within a fibroadenoma: a case report. Acta Radiol. 47(7):643-645

\section{Publisher's Note}

Springer Nature remains neutral with regard to jurisdictional claims in published maps and institutional affiliations.

\section{Submit your manuscript to a SpringerOpen ${ }^{\circ}$ journal and benefit from:}

- Convenient online submission

- Rigorous peer review

- Open access: articles freely available online

High visibility within the field

- Retaining the copyright to your article

Submit your next manuscript at $\boldsymbol{\nabla}$ springeropen.com 